\title{
Absolute Number Densities of Helium Metastable Atoms Determined by Atomic Absorption Spectroscopy in Helium Plasma-based Discharges Used as Ambient Desorption/Ionization Sources for Mass Spectrometry \\ ${ }^{1}$ Charlotte Reininger, ${ }^{1}$ Kellie Woodfield, ${ }^{2}$ Joel D. Keelor, ${ }^{2}$ Adam Kaylor, Facundo M. Fernández, ${ }^{1}$ Paul B. Farnsworth \\ ${ }^{1}$ Brigham Young University, Department of Chemistry and Biochemistry, Provo, UT 84602 \\ ${ }^{2}$ Georgia Institute of Technology, School of Chemistry and Biochemistry, Atlanta, GA 30332
}

\begin{abstract}
The absolute number densities of helium atoms in the $2{ }^{3} \mathrm{~S}_{1}$ metastable state were determined in four plasma-based ambient desorption/ionization sources by atomic absorption spectroscopy. The plasmas included a high-frequency dielectric barrier discharge (HF-DBD), a low temperature plasma (LTP), and two atmospheric-pressure glow discharges, one with AC excitation and the other with DC excitation. Peak densities in the luminous plumes downstream from the discharge capillaries of the HF-DBD and the LTP were $1.39 \times 10^{12} \mathrm{~cm}^{-3}$ and $0.011 \times$ $10^{12} \mathrm{~cm}^{-3}$, respectively. Neither glow discharge produced a visible afterglow, and no metastable atoms were detected downstream from the capillary exits. However, densities of $0.58 \times 10^{12}$ $\mathrm{cm}^{-3}$ and $0.97 \times 10^{12} \mathrm{~cm}^{-3}$ were measured in the interelectrode regions of the AC and DC glow discharges, respectively. Time-resolved measurements of metastable atom densities revealed significant random variations in the timing of pulsed absorption signals with respect to the voltage waveforms applied to the discharges.
\end{abstract}




\section{Introduction}

The introduction of desorption electrospray ionization (DESI) in 2004 [1] began a period of explosive growth in the development of ambient ionization sources for mass spectrometry. The most general name for these techniques, ambient desorption/ionization (ADI), serves as a reminder that the sources often perform two functions: removal of the analyte from the sample surface and ionization of the analyte for mass analysis. An impressive variety of ADI sources have been reported in the literature, as summarized in several reviews on the topic [2-6]. ADI sources can be loosely assigned to three categories: solvent-based sources, of which DESI is the prime example, plasma-based sources, including, for example, direct analysis in real time (DART) [7] and the low temperature plasma (LTP) [8], and hybrid sources, which separate the desorption and ionization functions of the source between distinct instrumental components and events.

This report focuses on the characterization of four plasma-based ionization sources, with the goal of identifying important commonalities and differences in their modes of operation. The four sources include two dielectric barrier discharges (DBDs), the LTP [8] and a second plasma that shares the LTP's coaxial electrode geometry, but with a smaller discharge tube, different electrode dimensions, and a much-higher operating frequency [9]. The remaining two discharges share a common point-to-disk electrode geometry, but one is operated with DC excitation and the other with AC excitation. The geometry is similar to the flowing atmospheric-pressure afterglow developed by the Hieftje group [10]. All four discharges were operated in pure helium as the plasma support gas. Helium is the most commonly used support gas for plasma ADI sources. Its appeal lies in the fact that sustaining a plasma in helium is relatively straightforward and that helium plasmas generate multiple long-lived species that have enough internal energy 
that they can, through collisions, ionize almost any neutral atom or molecule with which they come in contact. In addition, helium's high thermal conductivity facilitates analyte thermal desorption.

Analyte ionization in He plasma-based ADI sources is not a straightforward process. It is not a simple matter of energy transfer between an energetic He atom or ion and the analyte molecule. Rather, it almost certainly occurs by some variation of a sequence of reactions outlined by Dzidic, et al. [11] more than three decades ago for atmospheric pressure ionization sources, reproduced below as equations 1-6.

$$
\begin{gathered}
\mathrm{He}\left(1 \mathrm{~s}{ }^{1} \mathrm{~S}\right)+\mathrm{e}^{-} \rightarrow \mathrm{He}\left(2 \mathrm{~s}{ }^{3} \mathrm{~S}\right)+e^{-} \\
\mathrm{He}\left(2{ }^{3} \mathrm{~S}\right)+\mathrm{N}_{2} \rightarrow \mathrm{N}_{2}^{+}+\mathrm{He}\left(1 \mathrm{~s}{ }^{1} \mathrm{~S}\right)+\mathrm{e}^{-} \\
2 \mathrm{~N}_{2}+\mathrm{N}_{2}^{+} \rightarrow \mathrm{N}_{4}^{+}+\mathrm{N}_{2} \\
\mathrm{~N}_{4}^{+}+\mathrm{H}_{2} \mathrm{O} \rightarrow 2 \mathrm{~N}_{2}+\mathrm{H}_{2} 0^{\bullet+} \\
\mathrm{H}_{2} \mathrm{O}^{\bullet+}+\mathrm{nH}_{2} \mathrm{O} \rightarrow\left[\left(\mathrm{H}_{2} \mathrm{O}\right)_{\mathrm{n}}+\mathrm{H}^{+}+\mathrm{OH}^{\bullet}\right. \\
{\left[\left(\mathrm{H}_{2} \mathrm{O}\right)_{\mathrm{n}}+\mathrm{H}\right]^{+}+\mathrm{M} \rightarrow[\mathrm{M}+\mathrm{H}]^{+}+\mathrm{nH}_{2} \mathrm{O}}
\end{gathered}
$$

In the presence of a solvent or other concentrated matrix species, the sequence of reactions leading to analyte ionization may involve the matrix, as suggested by Song, et al. [12]. If a sequence of reactions such as 1-6 does control analyte ionization in helium plasma-based ADI sources, then one would expect to find a correlation between source sensitivity and the concentration of the limiting reagent in the sequence. Furthermore there should be a relationship between the concentration of the limiting reagent and the source's susceptibility to matrix effects 
and ionization suppression. Sources with a high concentration of the limiting reagent compared to the concentrations of ionizable species generated from the sample should perform better than those sources that generated a limited concentration of the ionizer (sometimes referred as "reactant ion(s)") compared to ionization targets in the sample. In the latter case, selective ionization of concomitant species can lead to severe suppression of analyte ionization [13].

It follows, then, that identification of the limiting reagent and measurement of its concentration should form a solid basis for source comparison and point the direction for the development of improved sources. In this paper we present the absolute number densities of He $(2 \mathrm{~s}){ }^{3} \mathrm{~S}_{1}$ metastable atoms, hereafter designated as $\mathrm{He}_{\mathrm{m}}$, determined by atomic absorption spectroscopy. We then compare the measured densities with calculated estimates of the densities of $\mathrm{N}_{2}$ and $\mathrm{H}_{2} \mathrm{O}$, identifying the $\mathrm{He}$ metastable atoms as the probable limiting reagent in the $\mathrm{He}$ plasma-based ADI sources studied here.

\section{Experimental}

\subsection{He plasma sources}

\subsubsection{High-frequency $D B D$}

A high-frequency dielectric barrier discharge (HF-DBD) is shown schematically in Fig. 1a and photographically in Fig. 1b. The discharge, similar to the one characterized in ref. [9], is notable for the luminous plume that extends approximately $30 \mathrm{~mm}$ downstream from the capillary exit. Ultra high purity helium (99.999\%) (Airgas, Radnor, PA, U.S.A.) was used in all of the experiments, and in this discharge flowed through the capillary at a rate of $2 \mathrm{~L} / \mathrm{min}$. The He flow rate was controlled by an MKS 1170A mass flow controller and an MKS 246C power supply/readout (MKS Instruments, Andover, MA, U.S.A.). AC power was supplied to the DBD 
with an ENI HPG-2 rf power supply (MKS Instruments, Andover, MA, U.S.A.). The optimized operating frequency was determined by minimizing the difference between the load and forward power, as reported on the front panel of the power supply. A $500 \mathrm{MHz}$ digital oscilloscope (LeCroy Corporation, Chestnut Ridge, NY, U.S.A.) was used to monitor the applied voltage waveform through a monitor output on the power supply. The power and frequency settings used for the experiment were $31 \mathrm{~W}$ and $350 \mathrm{kHz}$, respectively.

\subsubsection{LTP}

The LTP, shown in Fig. 2 was constructed based on a design developed by the Cooks group [8]. A lab-constructed power supply based on an automobile ignition coil supplied a 9.7 $\mathrm{kV}$ p-p voltage to the LTP at a frequency of $4.5 \mathrm{kHz}$. The He flow rate was $1 \mathrm{~L} \mathrm{~min}^{-1}$.

\subsubsection{Atmospheric-Pressure Glow discharges}

The two glow discharges used in the study are shown in Fig. 3. The discharges in Fig. 3b and Fig. 3c differ only in their mode of excitation. The electrode geometry and dimensions shown in Fig. 3a were the same for both sources. Gas exited the cell through a 1-mm-diameter hole in the end cap. A He flow of $2 \mathrm{~L} \mathrm{~min}^{-1}$ was used in both excitation modes. The cap electrode was grounded. A BHK 1000-0.2 MG high voltage power supply (KEPCO, Inc., Flushing, NY, U.S.A.) operated in current-limited mode at $25 \mathrm{~mA}$ powered the DC discharge with the tungsten pin serving as the cathode. At that current, the voltage applied to the pin was $494 \mathrm{~V}$. A $2.24 \mathrm{k} \Omega$ resistor in series with the discharge stabilized the circuit. In the AC mode the HPG-2 power supply that was used for the HF-DBD was used to apply high voltage to the pin electrode at a frequency of $325 \mathrm{kHz}$. The power delivered to the AC discharge was approximately $4 \mathrm{~W}$. 


\subsubsection{Low-pressure reference cell}

A low-pressure He glow discharge was constructed to provide an accurate frequency calibration axis for the atomic absorption measurements, based on the well-known wavelengths

of the He $2 \mathrm{~s}{ }^{3} \mathrm{~S}_{1}-2 \mathrm{p}{ }^{3} \mathrm{P}_{0}, 2 \mathrm{~s}{ }^{3} \mathrm{~S}_{1}-2 \mathrm{p}{ }^{3} \mathrm{P}_{1}$, and $2 \mathrm{~s}{ }^{3} \mathrm{~S}_{1}-2 \mathrm{p}{ }^{3} \mathrm{P}_{2}$ transitions at wavelengths of $1082.9091140 \mathrm{~nm}, 1083.0250105 \mathrm{~nm}, 1083.0339773 \mathrm{~nm}$, respectively [14]. The low pressure collisional broadening is reduced sufficiently that the peaks of the individual transitions could be accurately located, and He metastable atom densities were high enough in the positive column of the discharge to give excellent $\mathrm{s} / \mathrm{n}$ ratios in absorption measurements. The design for the lowpressure reference cell, illustrated in Fig. 4, was adapted from Weijian Lu et al. [15]. A glass to metal adaptor (stainless steel to 7740 Pyrex-UHV Series, MDC Vacuum Products LLC, Hayward, CA, U.S.A.) with an outer diameter of $12.7 \mathrm{~mm}$ served as the hollow cathode. A tungsten pin ( $2 \mathrm{~mm}$ dia.) served as the anode. Two quartz windows attached to the discharge tube near the anode provided a clear optical path through the positive column of the discharge. The discharge tube was mounted to a six-way stainless steel cross (MDC Vacuum Products LLC, Hayward, CA, U.S.A.), which was also used to connect the helium inlet, a pressure gauge (Granville Philips convection gauge, Longmont, CO, U.S.A.), a vacuum pump, and a 1.7 L stainless steel buffer reservoir. After baking and purging, the cell was operated at a pressure of 0.5 Torr and a current of $6 \mathrm{~mA}$ at $350 \mathrm{~V}$. With a clean fill the peak absorbances in the reference cell were as high as 0.7 .

\section{Atomic Absorption Measurements}

\subsection{Spectrometer}


The instrumentation used for absorption measurements is depicted schematically in Fig. 5. It is similar to a system used by Urabe, et al. [16] to study excitation mechanisms in an atmospheric-pressure DBD. The primary light source was a TLB-6900 VortexTM II laser (New Focus, Santa Clara, CA, U.S.A.) with a nominal wavelength of $1083 \mathrm{~nm}$ and sufficient mode hop free tuning range to cover all three of the He transitions listed in section 2.1.4. The specified linewidth of the laser is $<300 \mathrm{kHz}$. After the beam passed through an optical isolator (I-106T4L, Isowave, Dover, $\mathrm{NJ}$ ) beam splitters were used to pick off sample and reference beams, and the remainder of the beam was directed into a confocal Fabry-Perot interferometer, which was used as a frequency ruler.

The sample beam was focused and recollimated with a matched pair of achromats (50 mm f.1.). The plasma under study was positioned at the beam waist between the lenses. Sample and reference beams were monitored by matched detection channels, each of which consisted of an InGaAs photodiode (SM05PD5A, Thorlabs, Newton, NJ) and a differential amplifier (AM501, Tektronix, Beaverton, OR). The amplifier outputs were digitized with a multifunction I/O card (DAQ PCI-6110, National Instruments, Austin, TX), or passed to a digital oscilloscope (Wavesurfer, LeCroy, Chestnut Ridge, NY). For time-averaged absorbance measurements the laser was scanned back and forth across the wavelength range encompassing all three $\mathrm{He}$ transitions at a frequency of $40 \mathrm{mHz}$. For time-resolved measurements the laser was tuned to the blended peak of the two strongest, and the sample and reference intensities were recorded as a function of time. To speed the responses of the photodiodes for time-resolved measurements, they were terminated in $50 \mathrm{ohms}$ and the bandwidths of the amplifiers were set to their maximum values of $1 \mathrm{MHz}$. During all of the experiments the responses of the system were checked 
regularly with neutral density filters to ensure that neither the electronics nor the optical transitions were saturated by the laser.

Because an accurate laser frequency scale was essential for the calculation of number densities, we calibrated the frequency axis by simultaneously recording the output of the FabryPerot interferometer and absorption in the low-pressure reference cell. From the known spacing between the He lines and the number of fringes between peaks, we determined the free spectral range of the interferometer to be $730.1 \mathrm{MHz}$. In subsequent measurements on the analytical discharges, the interferometer fringes served as a precise frequency ruler.

\subsection{Absolute Number Density Calculations}

For a situation such as existed in our instrument, i.e. that the spectral bandwidth of the source was much narrower than the spectral width of the absorption transition, the absorbance for an individual transition is given by [17]:

$$
\mathrm{A}_{v \alpha}=\frac{\mathrm{A}_{21} \mathrm{c}^{2} \mathrm{~g}_{2 \alpha} \mathrm{S}_{v \alpha} \mathrm{n}_{1}}{v_{\alpha}^{2} \mathrm{~g}_{1} 8 \pi} \cdot \frac{\mathrm{x}}{\ln (10)}
$$

where $A_{v \alpha}$ is the frequency-dependent absorbance, $A_{21}$ is the Einstein A coefficient, $c$ is the speed of light, $g_{1}$ and $g_{2}$ are the degeneracies of the lower and upper levels, respectively, $S_{v}$ is the normalized line shape function, $n_{1}$ is the number density of absorbing atoms, and $x$ is the absorption path length. The subscript $\alpha$ designates a specific transition. In our experiments absorbances were measured over a narrow frequency range encompassing three partiallyresolved transitions, so the measured absorbance values are given by:

$$
A_{v \text { total }}=\frac{\mathrm{A}_{21} \mathrm{c}^{2} \mathrm{n}_{1}}{\mathrm{~g}_{1} 8 \pi} \cdot \frac{\mathrm{x}}{\ln (10)}\left(\frac{\mathrm{g}_{2 \alpha} \mathrm{S}_{v \alpha}}{v_{\alpha}^{2}}+\frac{\mathrm{g}_{2 \beta} \mathrm{S}_{v \beta}}{v_{\beta}^{2}}+\frac{\mathrm{g}_{2 \gamma} \mathrm{S}_{v \gamma}}{v_{\gamma}^{2}}\right)
$$


where the subscripts $\alpha, \beta$ and $\gamma$ refer to the three helium transitions. Note that the Einstein coefficients for the three transitions are identical, and are reported with an accuracy of better than $0.3 \%$ [18]. Integrating over the narrow frequency range scanned by the laser, considering the frequency terms to be constant, yields:

$$
\mathrm{A}_{\text {int }}=\frac{\mathrm{A}_{21} \mathrm{c}^{2} \mathrm{n}_{1}}{\mathrm{~g}_{1} 8 \pi} \cdot \frac{\mathrm{x}}{\ln (10)}\left(\frac{\mathrm{g}_{2 \alpha}}{v_{\alpha}^{2}}+\frac{\mathrm{g}_{2 \beta}}{v_{\beta}^{2}}+\frac{\mathrm{g}_{2 \gamma}}{v_{\gamma}^{2}}\right)
$$

Solving for $\mathrm{n}_{1}$ gives:

$$
\mathrm{n}_{1}=\frac{\mathrm{A}_{\mathrm{int}} \mathrm{g}_{1} 8 \pi}{\mathrm{A}_{21} \mathrm{c}^{2}} \cdot \frac{\ln (10)}{\mathrm{x}}\left(\frac{\mathrm{g}_{2 \alpha}}{v_{\alpha}^{2}}+\frac{\mathrm{g}_{2 \beta}}{v_{\beta}^{2}}+\frac{\mathrm{g}_{2 \gamma}}{v_{\gamma}^{2}}\right)^{-1}
$$

We calculated number densities for the $\mathrm{He}_{\mathrm{m}}$ atoms from equation 10 using numericallyintegrated absorbance values and path lengths equal to the inner diameters of the tubing used to generate the discharges. Note that, unlike Urabe, et al. [16], we did not attempt to radially resolve the density measurements across the inhomogeneous cross sections of the discharges. The reported values are line-of-sight averages.

\section{Results and discussion}

\subsection{HF-DBD results}

The HF-DBD is the source that we have studied most extensively in our lab so it served as a benchmark against which the other sources were compared. Representative wavelength scans of the HF-DBD and low-pressure reference source are presented in Fig. 6. The Gaussian profiles in the low-pressure discharge source are dominated by Doppler broadening. In contrast, the Voigt profiles in the atmospheric-pressure HF-DBD are dominated by collisional broadening. 
Note that the wings of the lines are still above baseline at both scan limits. For the data reported here, we did not extrapolate beyond the experimental data beyond the scan limits of the laser to include the extreme line wings in our number density calculations. For a limited data set, the experimental results were fit to Voigt profiles, and the integrated absorbances were determined with broader limits from the fitted curves. That exercise produced number densities that were on average 2.5 percent higher than those obtained by straight numerical integration of the experimental data. Our reported values are systematically $2-3$ percent low. Given larger errors imposed by the assumption that the absorption path lengths matched the internal diameters of the capillaries, the reported values include only the integrated experimental data without inclusion of contributions from the extreme line wings.

Densities were measured as a function of position downstream from the capillary exit, with results presented in Fig. 7. The shape of the plot is in good agreement with data for relative densities of $\mathrm{He}_{\mathrm{m}}$ atoms determined by laser-induced fluorescence and reported in reference [9]. The time-averaged value at $1.5 \mathrm{~mm}$ downstream from the capillary tip $\left(1.4 \times 10^{12} \mathrm{~cm}^{-3}\right)$ is significantly higher than the values reported for other atmospheric-pressure plasmas. Urabe, et al. measured a peak, time-resolved density of $4.5 \times 10^{11} \mathrm{~cm}^{-3}$ in a DBD sustained in a 4-mmdiameter discharge tube [16]. Niermann, et al. reported densities of $1.4 \times 10^{11} \mathrm{~cm}^{-3}$ near the electrodes of a radiofrequency microplasma sustained in helium [19].

The detection limit for the time-averaged measurements with a 1-mm pathlength was approximately $3 \times 10^{9} \mathrm{~cm}^{-3}$. This estimate was based on averaging densities from 24 individual scans across the wavelength range covered in Fig. 6 to produce a signal three times the standard deviation of the blank.

\subsection{Comparative results for four plasmas}


Absolute number densities for the four plasmas described in section 2.1 are summarized in Table 1. Several features of the table are notable. The HF-DBD produces the highest density of $\mathrm{He}_{\mathrm{m}}$ atoms. This result is not surprising, given the fact that it consumes the highest power among the sources, and does so in the smallest dimensions. The lower numbers from the LTP can also be easily rationalized in terms of duty cycle, as will be discussed in more detail in the section on time-resolved measurements. The $\mathrm{He}_{\mathrm{m}}$ densities in the glow discharges were below detection limits downstream from the end cap electrode, irrespective of excitation mode. Given the significant densities detected in the interelectrode region, this result points to a highly efficient quenching mechanism for the metastable atoms as the gas flows out through the end cap electrode. Reaction 2, Penning ionization of $\mathrm{N}_{2}$ by $\mathrm{He}$, is a likely candidate. Definitive identification of the quenching mechanism will require measurement of possible reaction products downstream from the end cap exit. The difference between the DC and AC modes is consistent with simulations of lower-pressure discharges by Boegaerts and Gijbels [20]. They reported higher densities of argon metastable atoms in the interelectrode region with RF excitation than were calculated with DC excitation.

It is worth noting that in the application of a plasma as an ADI source, the important quantity in determining source performance may not be the concentration of reagent ions, but rather the total flux of those ions delivered to the sample, depending on how large a volume of gas is sampled by the mass spectrometer. In cases where the total flux of reagent ions dictates source performance, the product of the reagent ion density and the volume in which it produced is the relevant quantity, and the $\mathrm{AC}$ glow discharge looks particularly promising. The density of $\mathrm{He}_{\mathrm{m}}$ generated in the AC glow discharge is only about 40 percent of that generated in the HFDBD, but the cross sectional area of the glow discharge is 16 time that of the HF-DBD. 
Ultimately the relative efficacies of the sources should depend on the densities of ionizers that interact with the sample, and the determination of the He number densities is only a first step in characterizing the production of the key ionizing species.

\subsection{Time-resolved density measurements}

\subsubsection{HF-DBD}

The densities reported in Table 1 are time averages, which are appropriate for assessing the potential of the plasmas as ADI sources, but which mask important information about the mechanisms by which the discharges operate. Three of the four plasma are driven by AC waveforms, and it is well known that the DBD plasmas produce a series of plasma "bullets" synchronous with the voltage waveform [21-24]. Because the determination of absolute number densities required scanning of the laser over the entire widths of the transitions, such determinations were not practical in a time-resolved mode. Rather, as described in the experimental section, we measured time resolved relative number densities by tuning the laser to the largest absorption peak and then monitoring the transmitted intensity as a function of time. The results for the HF-DBD, recorded $0.5 \mathrm{~mm}$ downstream from the exit of the capillary, are presented in Fig. 8. These results are surprising because they indicate that the plasma bullets are being generated at half the frequency of the voltage waveform, with occasional exceptions, as shown in the last cycle in Fig. 8. We are not aware of any other reports in the literature of this phenomenon, and the observed behavior may be unique to our combination of electrode geometry and operating frequency. It is also possible that this type of behavior would pass unnoticed because many measurements of the bullet behavior involve either ensemble averaging or stroboscopic imaging, both of which would obscure the erratic every-other-cycle behavior evident in fig. 8. Because of the occasional single-cycle gap between bullets, any averaging 
synchronized with the voltage waveform gives the impression of modulation at the voltage frequency, with half the true depth of modulation. Previous measurements on the HF-DBD suffered from this artifact [9]. The rise times of the absorption signals are limited by the bandwidths of the amplifiers. The fall times are close to the period of the voltage waveform, 3-4 $\mu$ sec.

The frequency for the HF-DBD operation was selected to maximize power coupling between the power supply and the plasma. Tuning away from the optimum frequency produced patterns more erratic than that shown in fig. 8 , but none that consistently produced an absorption maximum with every cycle of the voltage waveform. At half the optimum frequency the plasma cannot be sustained. From the perspective of operating the HF-DBD as an ADI source, the observed time-resolved behavior is a curiosity that has little impact on source performance. On a long-term basis the plasma is exceptionally stable. From the perspective of understanding fundamental DBD mechanisms, the time-resolved behavior clearly deserves additional in-depth study that is beyond the scope of this report.

\subsubsection{LTP}

Time-resolved absorbances for the LTP are presented in Fig. 9. As was the case with the HF-DBD, the synchronization of the pulses of high He metastable density with the voltage is imperfect. A small pulse occurs regularly in the rising portion of the negative half cycle in the voltage waveform. Larger events in the form of 1-3 pulses of varying amplitude occur following the peak in the positive half cycle. As was the case with the HF-DBD, the pulse rise times are limited by the amplifier bandwidths. The pulse fall times in the LTP are longer than they were in the HF-DBD, approximately $6 \mu$ sec. The difference in fall times may reflect the different dimensions of the two discharges. 
The duty cycle in the LTP is much lower than it is in the HF-DBD, a difference that is reflected in the relatively low time-averaged $\mathrm{He}_{\mathrm{m}}$ densities observed in the LTP. Simply from the perspective of producing high densities of potential ionizers, high frequencies and powers are advantageous in dielectric barrier discharge sources.

\subsubsection{Glow discharges}

The time-resolved results from the glow discharges were unexceptional. The DC glow discharge generated a low, constant density of $\mathrm{He}_{\mathrm{m}}$ atoms at a location half way between the two electrodes. The AC glow discharge, as shown in fig. 10, generated a high, steady-state density of metastable atoms, upon which fluctuations at the frequency of the voltage waveform were superimposed. There was no evidence of the kind of erratic behavior that was observed in the two dielectric barrier discharges.

\section{Limiting reagent}

A natural concern raised by the term "ambient" ionization is that ambient conditions are, by definition, uncontrolled. Under ambient conditions the densities of some of the key species in reactions 1-6 can vary significantly from lab to lab. For example, the atmospheric pressure at our location is 85 percent of the pressure in a lab at sea level. The density of water vapor can vary by an order of magnitude. However, the numbers reported in table 1 combined with simple ideal gas calculations, put any concerns in perspective. At a pressure of one atmosphere and a temperature of $298 \mathrm{~K}$, the number density of an ideal gas is $2.5 \times 10^{19} \mathrm{~cm}^{-3}$. Gas temperatures in the gas jets emerging from the plasmas are relatively low. Reported rotational temperatures are between $250 \mathrm{~K}$ and $550 \mathrm{~K}$ [25]. Gas densities in the discharges are approximately $10^{19} \mathrm{~cm}^{-3}$, which means that the helium metastable atoms are present at the ppm level, close to the levels of 
impurities in the high-purity helium typically used as a support gas for the discharges. Both $\mathrm{N}_{2}$ $\left(\sim 2 \times 10^{19} \mathrm{~cm}^{-3}\right)$ and $\mathrm{H}_{2} \mathrm{O}\left(\sim 2 \times 10^{17} \mathrm{~cm}^{-3}\right.$ at 30 percent relative humidity $)$ are present in room temperature air in such large excess that changes in atmospheric pressure and humidity are irrelevant. The principal factors affecting the rates and extent of completion of reactions 2-6 are the purity of the support gas and the flow patterns of the helium jet as it mixes with air [26]. In the sequence suggested by equations $1-6$, the $\mathrm{He}_{\mathrm{m}}$ atoms are clearly the limiting reagent.

\section{Summary and Conclusions}

The four helium-based ADI sources examined in this study produced dramatically different results, as determined by the densities of $\mathrm{He}_{\mathrm{m}}$ atoms in the exit plumes of the discharges. Two dielectric barrier discharges, the LTP and the HF-DBD were similar in that they generated bursts of $\mathrm{He}_{\mathrm{m}}$ atoms that extended downstream from exit of the capillary containing the discharge. The HF-DBD generated much higher average $\mathrm{He}_{\mathrm{m}}$ number densities than did the LTP, primarily due a higher duty cycle in the operation of the plasma. Neither the AC nor DC glow discharge generated detectable $\mathrm{He}_{\mathrm{m}}$ atom densities outside the interelectrode region. Between the electrodes the $\mathrm{AC}$ generated densities $\sim 6$ times higher than the $\mathrm{DC}$ discharge. The absence of detectable afterglow in the glow discharges raises questions about the fate of the metastable atoms produced in those discharges that will only be answered by measurement of non-emitting species produced in reactions 2-6.

The long-term goal of our research is the development of improved ADI plasma sources for mass spectrometry. The next step in that process is the correlation of the fundamental optical performance reported here with ADI ion source mass spectrometric performance. Those measurements are in progress and will be reported in a separate publication. 
Acknowledgement This research was supported in part by the Chemical Sciences, Geosciences and Biosciences Division, Office of Basic Energy Sciences, Office of Science, U.S. Department of Energy. JDK and FMF are supported by the NSF and NASA Astrobiology Program under the NSF Center for Chemical Evolution (CHE-1004570). AK was supported by the US

Pharmacopeial Convention in the form of a Global Fellowship. 


\section{References}

[1] Z. Takats, J.M. Wiseman, B. Gologan, R.G. Cooks, Mass Spectrometry Sampling Under Ambient Conditions with Desorption Electrospray Ionization, Science, 306 (2004) 471-473.

[2] R. Alberici, R. Simas, G. Sanvido, W. Romão, P. Lalli, M. Benassi, I. Cunha, M. Eberlin, Ambient mass spectrometry: bringing MS into the "real world", Analytical and Bioanalytical Chemistry, 398 (2010) 265-294.

[3] D.R. Ifa, C.P. Wu, Z. Ouyang, R.G. Cooks, Desorption electrospray ionization and other ambient ionization methods: current progress and preview, Analyst, 135 (2010) 669-681.

[4] A. Venter, M. Nefliu, R. Graham Cooks, Ambient desorption ionization mass spectrometry, TrAC, Trends Anal. Chem., 27 (2008) 284-290.

[5] A.R. Venter, K.A. Douglass, J.T. Shelley, G. Hasman, E. Honarvar, Mechanisms of RealTime, Proximal Sample Processing during Ambient Ionization Mass Spectrometry, Anal. Chem., 86 (2014) 233-249.

[6] M.E. Monge, G.A. Harris, P. Dwivedi, F.M. Fernandez, Mass Spectrometry: Recent Advances in Direct Open Air Surface Sampling/Ionization, Chem. Rev., 113 (2013) 2269-2308.

[7] R.B. Cody, J.A. Laramee, H.D. Durst, Versatile New Ion Source for the Analysis of Materials in Open Air under Ambient Conditions, Anal. Chem., 77 (2005) 2297-2302.

[8] J.D. Harper, N.A. Charipar, C.C. Mulligan, X. Zhang, R.G. Cooks, Z. Ouyang, LowTemperature Plasma Probe for Ambient Desorption Ionization, Anal. Chem., 80 (2008) 90979104.

[9] M.S. Heywood, N. Taylor, P.B. Farnsworth, Measurement of Helium Metastable Atom Densities in a Plasma-Based Ambient Ionization Source, Anal. Chem., 83 (2011) 6493-6499. 
[10] F.J. Andrade, J.T. Shelley, W.C. Wetzel, M.R. Webb, G. Gamez, S.J. Ray, G.M. Hieftje, Atmospheric Pressure Chemical Ionization Source. 1. Ionization of Compounds in the Gas Phase, Anal. Chem., 80 (2008) 2646-2653.

[11] I. Dzidic, D.I. Carroll, R.N. Stillwell, E.C. Horning, Comparison of Positive-Ions Formed in Nickel-63 and Corona Discharge Ion Sources Using Nitrogen, Argon, Isobutane, Ammonia and Nitric-Oxide as Reagents in Atmospheric-Pressure Ionization Mass-Spectrometry, Anal. Chem., 48 (1976) 1763-1768.

[12] L.G. Song, S.C. Gibson, D. Bhandari, K.D. Cook, J.E. Bartmess, Ionization Mechanism of Positive-Ion Direct Analysis in Real Time: A Transient Microenvironment Concept, Anal. Chem., 81 (2009) 10080-10088.

[13] G.A. Harris, C.E. Falcone, F.M. Fernandez, Sensitivity "Hot Spots" in the Direct Analysis in Real Time Mass Spectrometry of Nerve Agent Simulants, J. Am. Soc. Mass. Spectrom., 23 (2012) 153-161.

[14] Y. Ralchenko, A.E. Kramida, J. Reader, N.A.T. (2011), NIST Atomic Spectra Database (Ver. 4.1.0), in, National Institute of Standards and Technology, Gaithersburg, MD, 2011. [15] W.J. Lu, D. Milic, M.D. Hoogerland, M. Jacka, K.G.H. Baldwin, S.J. Buckman, A practical direct current discharge helium absorption cell for laser frequency locking at $1083 \mathrm{~nm}$, Rev. Sci. Instrum., 67 (1996) 3003-3004.

[16] K. Urabe, T. Morita, K. Tachibana, B.N. Ganguly, Investigation of discharge mechanisms in helium plasma jet at atmospheric pressure by laser spectroscopic measurements, J. Phys. D: Appl. Phys., 43 (2010) 095201.

[17] J.D. Ingle, S.R. Crouch, Spectrochemical analysis, Prentice Hall, Englewood Cliffs, N.J., 1988. 
[18] W.L. Wiese, J.R. Fuhr, Accurate Atomic Transition Probabilities for Hydrogen, Helium, and Lithium, J. Phys. Chem. Ref. Data, 38 (2009) 565-726.

[19] B. Niermann, M. Boke, N. Sadeghi, J. Winter, Space resolved density measurements of argon and helium metastable atoms in radio-frequency generated He-Ar micro-plasmas, European Physical Journal D, 60 (2010) 489-495.

[20] A. Bogaerts, R. Gijbels, Description of the argon-excited levels in a radio-frequency and direct current glow discharge, Spectrochim. Acta, Part B, 55 (2000) 263-278.

[21] G.V. Naidis, Modelling of plasma bullet propagation along a helium jet in ambient air, Journal of Physics D-Applied Physics, 44 (2011).

[22] J. Jarrige, M. Laroussi, E. Karakas, Formation and dynamics of plasma bullets in a nonthermal plasma jet: influence of the high-voltage parameters on the plume characteristics, Plasma Sources Science \& Technology, 19 (2010).

[23] J. Shi, F. Zhong, J. Zhang, D.W. Liu, M.G. Kong, A hypersonic plasma bullet train traveling in an atmospheric dielectric-barrier discharge jet, Physics of Plasmas, 15 (2008) 013504-013505. [24] M. Teschke, J. Kedzierski, E.G. Finantu-Dinu, D. Korzec, J. Engemann, High-speed photographs of a dielectric barrier atmospheric pressure plasma jet, Plasma Science, IEEE Transactions on, 33 (2005) 310-311.

[25] G.C.Y. Chan, J.T. Shelley, J.S. Wiley, C. Engelhard, A.U. Jackson, R.G. Cooks, G.M. Hieftje, Elucidation of Reaction Mechanisms Responsible for Afterglow and Reagent-Ion Formation in the Low-Temperature Plasma Probe Ambient Ionization Source, Anal. Chem., 83 (2011) 3675-3686. 
[26] G.A. Harris, F.M. Fernandez, Simulations and Experimental Investigation of Atmospheric Transport in an Ambient Metastable-Induced Chemical Ionization Source, Anal. Chem., 81 (2009) 322-329. 


\begin{tabular}{|c|c|c|c|c|}
\hline Plasma Source & $\begin{array}{l}\text { Excitation } \\
\text { Mode }\end{array}$ & Location & $\begin{array}{c}\text { Path } \\
\text { Length } \\
(\mathrm{mm}) \\
\end{array}$ & $\begin{array}{c}\text { Density of } \mathrm{He} \\
(2 \mathrm{~s}){ }^{3} \mathrm{~S}_{1} \text { atoms } \\
\left(10^{12} \mathrm{~cm}^{-3}\right)\end{array}$ \\
\hline HF-DBD & $\mathrm{AC}, 350 \mathrm{kHz}$ & $\begin{array}{l}1 \mathrm{~mm} \text { downstream from } \\
\text { capillary exit }\end{array}$ & 1 & $1.39 \pm 0.03$ \\
\hline LTP & $\mathrm{AC}, 4.5 \mathrm{kHz}$ & $\begin{array}{c}1 \mathrm{~mm} \text { downstream from } \\
\text { capillary exit }\end{array}$ & 4 & $\begin{array}{c}0.01111 \pm \\
0.0007 \\
\end{array}$ \\
\hline Glow discharge & $\mathrm{AC}, 325 \mathrm{kHz}$ & $\begin{array}{c}1 \mathrm{~mm} \text { downstream from } \\
\text { end cap }\end{array}$ & $\sim 1$ & $<0.003$ \\
\hline Glow discharge & $\mathrm{AC}, 325 \mathrm{kHz}$ & $\begin{array}{c}\text { Midway between pin and } \\
\text { end cap electrodes }\end{array}$ & 4 & $0.585 \pm 0.006$ \\
\hline Glow discharge & $\mathrm{DC}$ & $\begin{array}{c}1 \mathrm{~mm} \text { downstream from } \\
\text { end cap }\end{array}$ & $\sim 1$ & $<0.003$ \\
\hline Glow discharge & $\mathrm{DC}$ & $\begin{array}{c}\text { Midway between pin and } \\
\text { end cap electrodes }\end{array}$ & 4 & $0.097 \pm 0.002$ \\
\hline
\end{tabular}

Table 1. Number densities of $\mathrm{He}_{\mathrm{m}}$ atoms for four plasma sources. 
Figure 1 HF-DBD: (a) schematic, (b) photograph

Figure 2 LTP: (a) schematic, (b) photograph

Figure 3 Glow discharges. (a) schematic, (b) photograph with DC excitation, (c) photograph with AC excitation.

Figure 4 Schematic of low-pressure hollow cathode reference discharge

Figure 5 Schematic of atomic absorption instrument. The low-pressure discharge was only in place during wavelength calibration measurements.

Figure 6 Absorption spectra for the HF-DBD (bold trace) and low-pressure reference discharge (gray trace).

Figure 7 Absolute number densities in the HF-DBD as a function of position downstream from the capillary exit. The error bars are the standard deviations of 24 replicate determinations made in rapid succession.

Figure 8 Time-resolved absorbance measured in the HF-DBD $0.5 \mathrm{~mm}$ downstream from the capillary exit. The bold trace is absorbance and the dashed trace is the voltage waveform from the power supply. Note that the rise times of the absorbance peaks are limited by the $1 \mathrm{MHz}$ bandwidth of the recording electronics.

Figure 9 Time-resolved absorbance measured in the LTP $0.5 \mathrm{~mm}$ downstream from the capillary exit. The bold trace is absorbance and the dashed trace is the voltage waveform from the power supply. 
Figure 10 Time-resolved absorbance measured in the AC glow discharge midway between the electrodes. The bold trace is absorbance and the dashed trace is the voltage waveform from the power supply. 
a

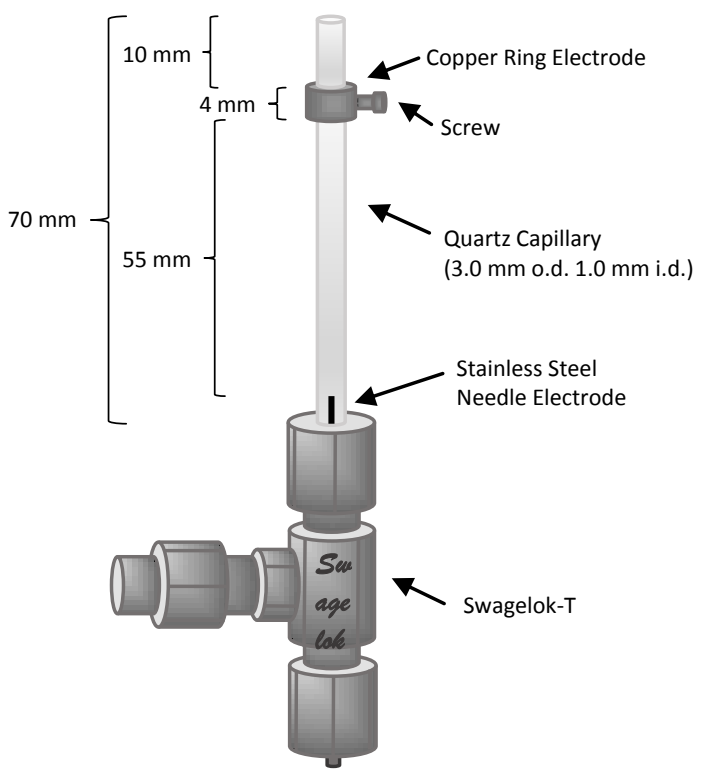

b

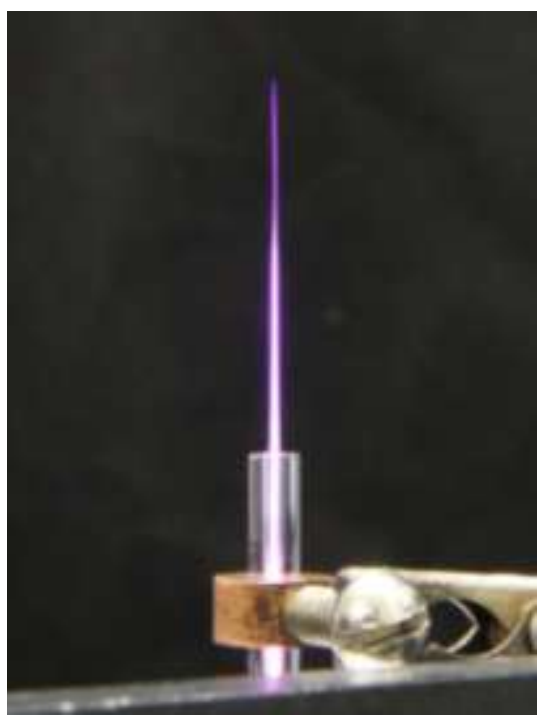

Figure 1 
a

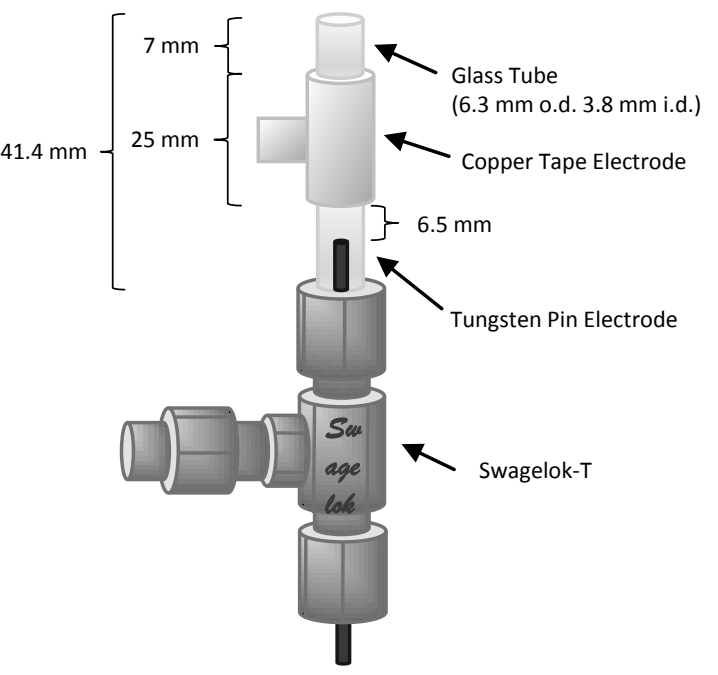

b

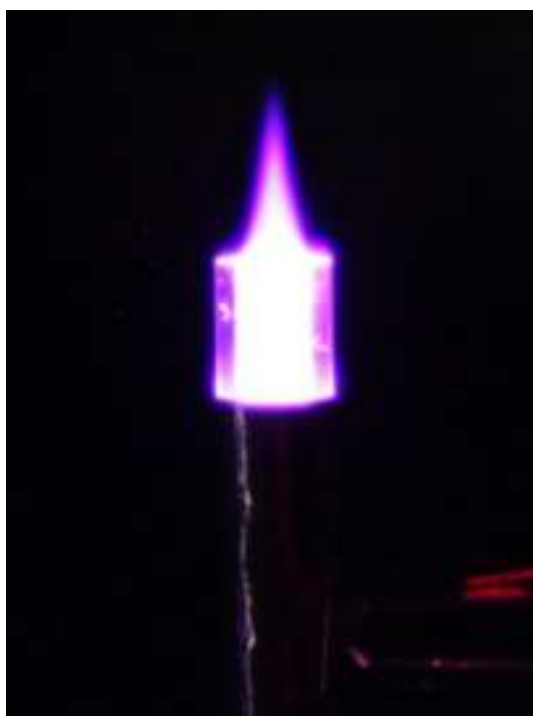

Figure 2 


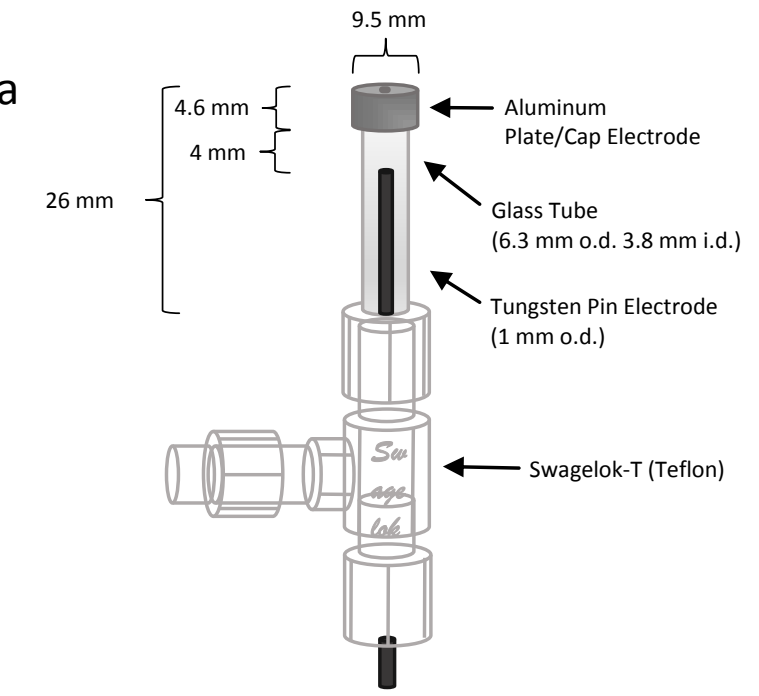

b

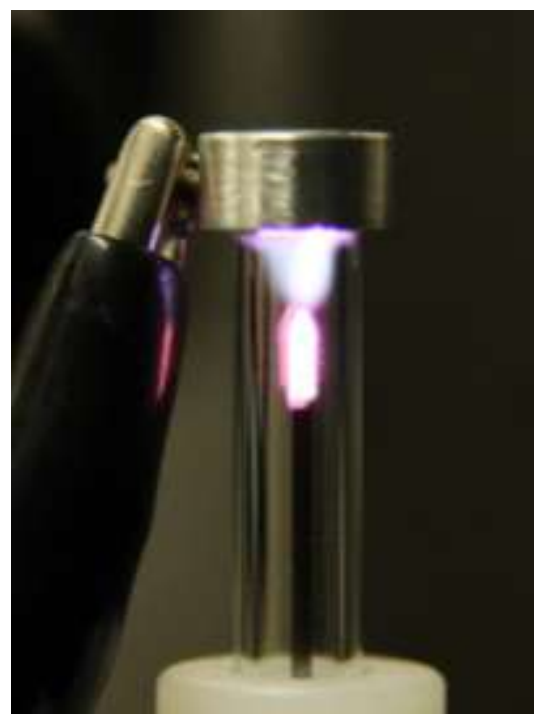

C

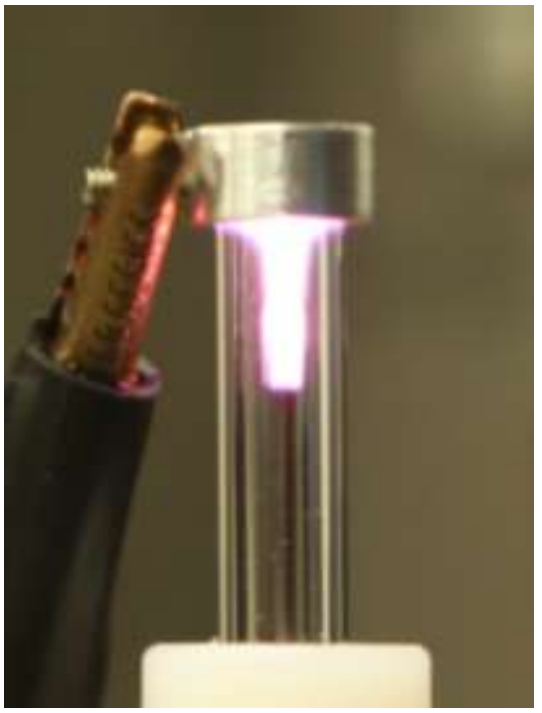

Figure 3 


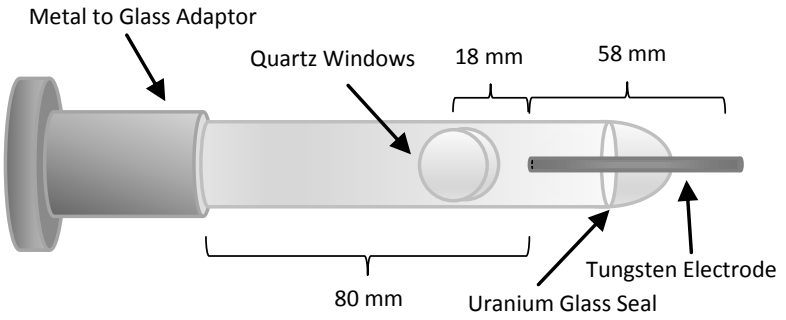

Figure 4 


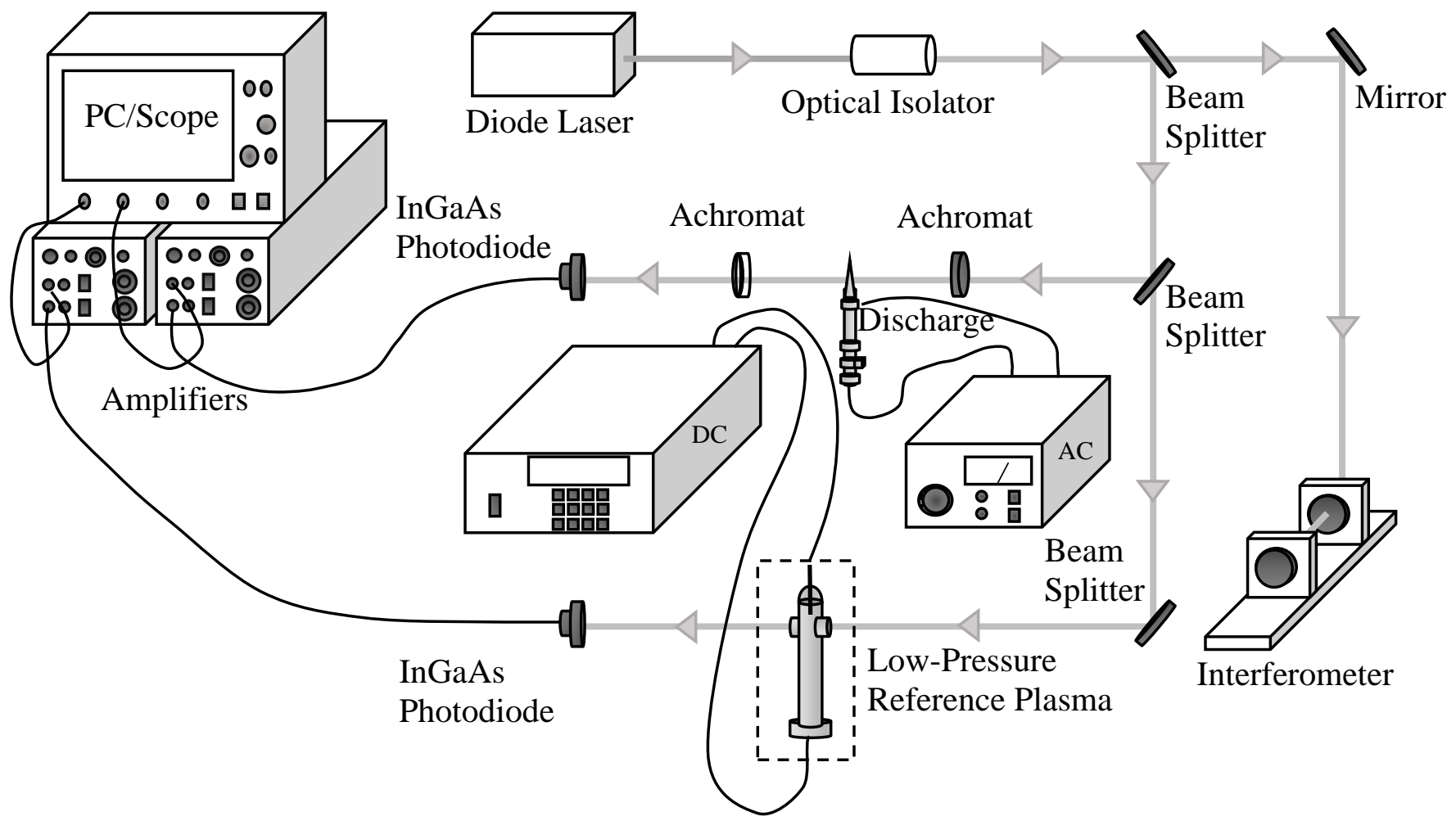

Figure 5 


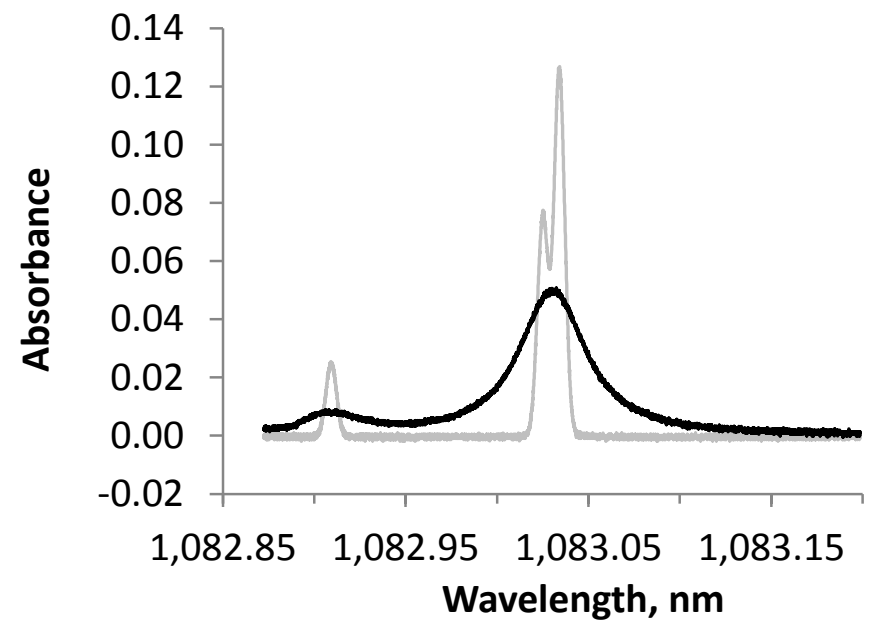

Figure 6 


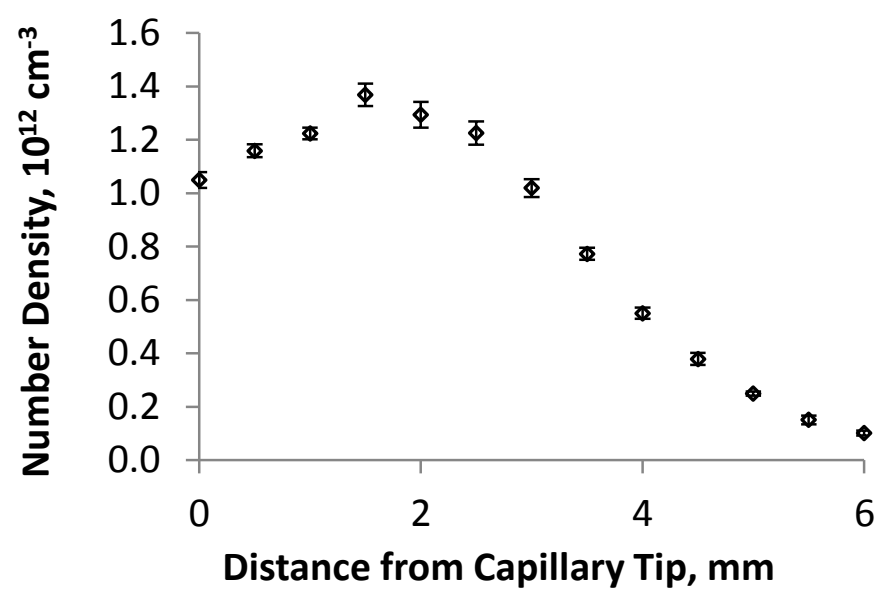

Figure 7 


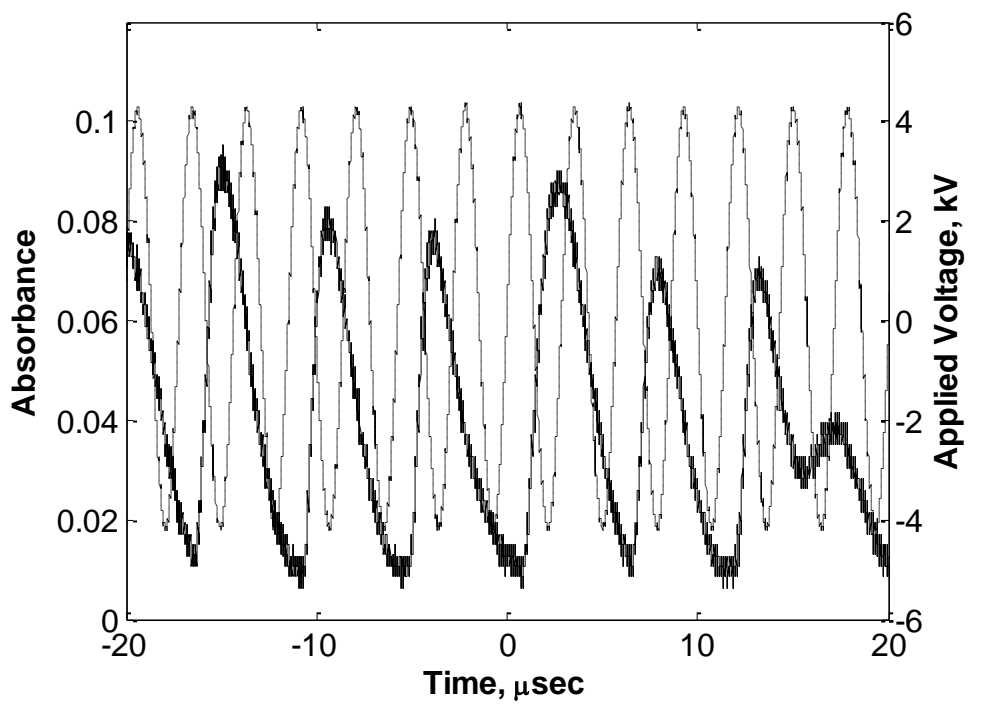

Figure 8 


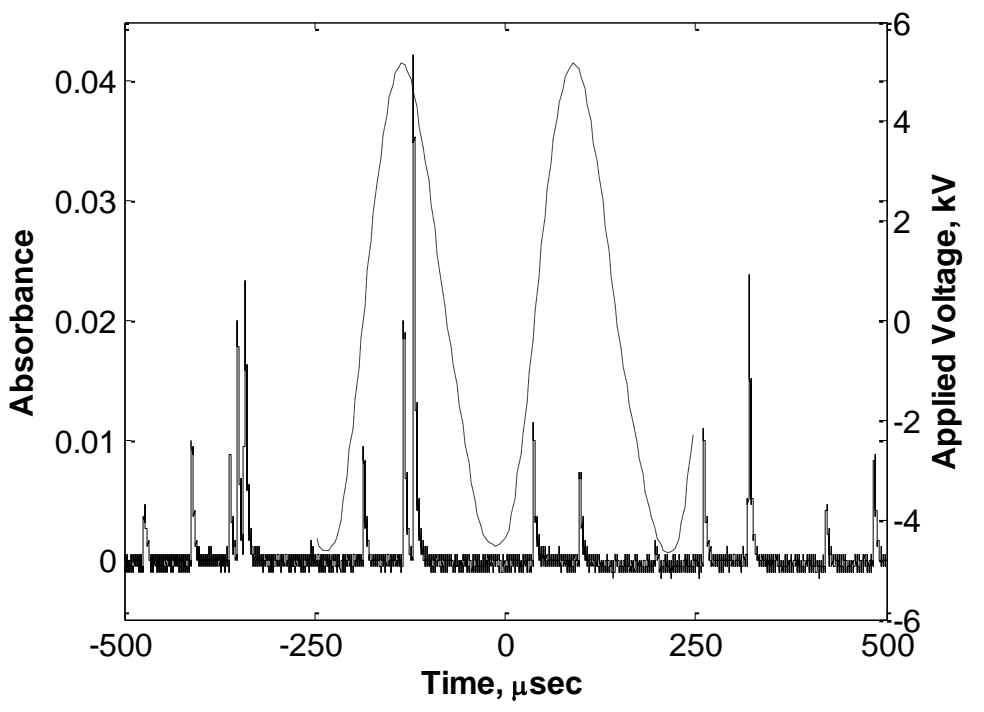

Figure 9 


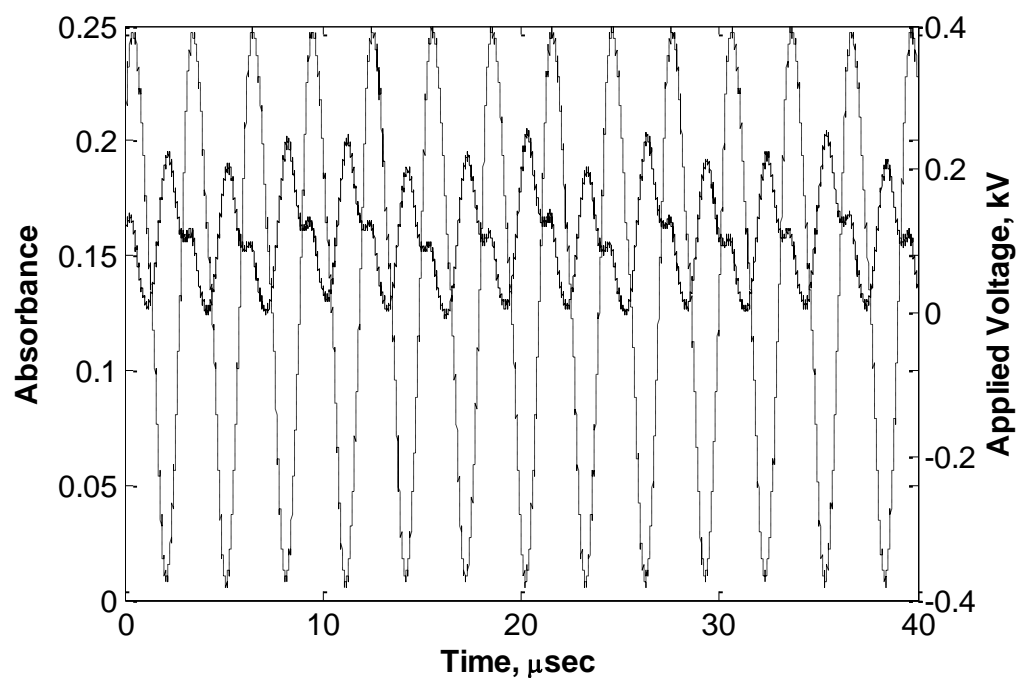

Figure 10 